\title{
Pró-Saúde e PET-Saúde como espaços de educação interprofissional
}

\author{
Marcelo Viana da Costa(a) \\ Karina Pavão Patrício(b) \\ Ana Maria Chagas Sette Câmara ${ }^{(c)}$ \\ George Dantas Azevedo(d) \\ Sylvia Helena Souza da Silva Batista(e)
}

Costa MV, Patrício KP, Câmara AMCS, Azevedo GD, Batista SHSS. Pro-Health

and PET-Health as interprofessional education spaces. Interface (Botucatu). 2015;

19 supl 1:709-20.

This paper aims to reflect on the National Reorientation Program for Health Professional Education (Pro-Health), as articulated with the Education by Work for Health Program (PET-Health); this was the main mobilizing set for interprofessional education adoption based on the challenges identified in the higher education institutions (HEI) that participated in PROPET. The data were obtained from reports (educational institutions and advisers of the Ministry of Health) using the FORMSUS platform. The data analysis consisted of a quantitative systematization and content analysis. It was emerged as themes: concepts and practices in interprofessional education: multi or inter? Interprofessional education and PROPET: unveiling potencies. The analysis indicated that PROPET induced new ways of interaction between the courses and their agents that were involved in real-life scenarios and identified important key points of health education, such as the articulation between education/health service and qualifications for teamwork.

Keywords: Health education. Teaching care integration services. Public health policy.
Este artigo pretende refletir sobre o Programa Nacional de Reorientação da Formação Profissional em Saúde (PróSaúde), articulado ao Programa de Educação pelo Trabalho para a Saúde (PET-Saúde) como cenário mobilizador para a adoção da educação interprofissional a partir dos desafios identificados nas instituições de Ensino Superior (IES) pelos participantes do PROPET. Os dados foram produzidos a partir de relatórios das IES e de assessores do Ministério da Saúde, via plataforma FORMSUS. A análise dos dados consistiu de uma sistematização quantitativa e análise de conteúdo. Emergiram como temas: concepções e práticas na educação interprofissional: multi ou inter? Educação Interprofissional e PROPET: desvelando potências. A análise indica que o PROPET vem induzindo novas formas de interação entre cursos envolvidos e seus atores, em cenários reais, enfrentando importantes pontos nevrálgicos da formação em saúde: a articulação ensino/serviço e a qualificação para o trabalho em equipe.

Palavras-chave: Educação em saúde. Serviços de integração docente-assistencial. Políticas públicas de saúde. (a) Departamento de Enfermagem, Campus Avançado Profa. Maria Elisa de Albuquerque Maia, Universidade do Estado do Rio Grande do Norte. BR 405 Km 3, Arizona. Pau dos Ferros, RN, Brasil. 59900-000. vianacostam@yahoo.com.br

(b) Departamento de Saúde Pública, Faculdade de Medicina de Botucatu, UNESP - Univ Estadual Paulista. Botucatu, SP, Brasil.pavao@fmb.unesp.br (c) Departamento de Fisioterapia, Universidade Federal de Minas Gerais. Belo Horizonte, MG, Brasil. anasettecamara@gmail.com

(d) Departamento de Morfologia, Centro de Biociências e Escola Multicampi de Ciências Médicas, Universidade Federal do Rio Grande do Norte. Natal, RN, Brasil.georgedantas.faimer@gmail.com

(e) Departamento Saúde, Educação e Sociedade, Universidade Federal de São Paulo, campus Baixada Santista. Santos,

SP, Brasil.sylvia.batista@unifesp.br 


\section{Introdução}

No contexto atual, é cada vez mais necessário pensar que mudanças significativas na realidade dos serviços de saúde - capazes de provocar maiores impactos nas condições gerais de vida e saúde das pessoas - somente serão possíveis frente a um processo de reforma do modelo de formação da força de trabalho em saúde ${ }^{1-3}$.

Um dos pontos de fragilidade do processo de formação dos profissionais de saúde é a pouca capacidade na formação de profissionais aptos ao efetivo trabalho em equipe, reproduzindo um modelo de atenção à saúde muito fragmentado e pouco resolutivo. Nesse sentido, alguns esforços para fortalecer essa discussão emergem no cenário global. Em 1988, por exemplo, foi apresentado o documento "Learning Together to work together for health", que deu início a uma série de iniciativas e articulações voltadas para a necessidade de se formarem profissionais mais aptos ao trabalho colaborativo nas equipes de saúde, a partir da lógica da educação interprofissional (EIP)4.

As ações e movimentos disparados pela "The network towards unity for health" também têm contribuído para a disseminação dos fundamentos da EIP, propondo que o termo multiprofissional seja substituído por interprofissional, para enfatizar a importância de serem criadas e implementadas situações de aprendizagem "com, para e sobre" diferentes profissões da saúde.

Recentemente, esse debate ganhou uma produção teórica importante, e que tem contribuído para retomar e fortalecer as reflexões sobre o processo de formação em saúde: o relatório produzido pela Comissão de Educação da Lancet ${ }^{1,5}$.

O relatório traz, com muita propriedade, os atuais problemas enfrentados pelos países na configuração dos serviços de saúde, e os desafios a serem assumidos pela educação dos profissionais de saúde. Essa assertiva impõe importantes desafios quando a realidade brasileira é posta em discussão. No Brasil, há importantes dispositivos de luta pela consolidação e fortalecimento do Sistema Único de Saúde (SUS), a partir dos pressupostos da reforma sanitária brasileira, que colocam como pontos centrais do sistema ${ }^{6}$ : o atendimento das necessidades de saúde da população, ênfase na transformação dos perfis epidemiológicos e mudanças do modelo assistencial.

Nesse sentido, as políticas de promoção de processos de mudanças na formação dos profissionais de saúde no Brasil têm assumido papel importante frente a esses desafios. As diretrizes curriculares nacionais dos cursos da área da saúde conseguem expressar a intenção de formar um novo perfil profissional, capaz de assumir seu importante papel na mudança das condições gerais de vida e saúde da população brasileira ${ }^{7}$.

Outro ponto que merece destaque, no contexto brasileiro, foi a criação da Secretaria de Gestão do Trabalho e da Educação em Saúde - SGTES, que fortaleceu o debate sobre a necessidade de reorientação da formação profissional, promovendo uma abordagem integral do processo saúdedoença, valorizando a atenção primária à saúde e integração entre as Instituições de Ensino Superior (IES) e os serviços públicos, na busca de fortalecer o SUS?.

No entanto, há uma série de problemas complexos que vão além da reestruturação curricular, como: inovação das estratégias de ensino e de aprendizagem; fortalecimento das relações ensino/ serviço na perspectiva da coparticipação em ambos os processos; redefinição dos papéis dos profissionais de saúde; fortalecimento do trabalho em equipe a partir da lógica colaborativa; e resgate da responsabilidade social dos profissionais em formação sobre o contexto em que se inserem ${ }^{9}$.

O contexto atual do processo de formação em saúde fortalece a lógica da separação das áreas do conhecimento, constituindo as 'tribos profissionais'10, estabelecendo áreas que podem/devem ou não dialogar - realidade muito presente nos departamentos acadêmicos. Os silos profissionais se fortalecem como estratégia para assegurar as identidades profissionais, com fortes implicações para o trabalho em saúde ${ }^{11}$, até mesmo negando sua essência: o trabalho colaborativo e compartilhado.

Diante das problemáticas relatadas, a EIP tem sido discutida como importante abordagem para a efetivação de mudanças das práticas profissionais, e, consequentemente, do modelo assistencial. A EIP se mostra necessária, na realidade brasileira, na medida em que busca qualificar os profissionais, gestores e instituições para o trabalho colaborativo em saúde, fundamental para a integralidade do cuidado ${ }^{12}$. 
Sendo assim, a perspectiva da EIP e do trabalho colaborativo, e suas bases teóricas e metodológicas, garantem aderência aos princípios da reforma sanitária brasileira, na medida em que apresentam elementos para mudanças importantes na dinâmica de produção dos serviços de saúde, a partir da ressignificação dos papéis profissionais, bem como de todos os atores envolvidos. Outrossim, permite (re)situar a figura do usuário e de suas necessidades, na centralidade do processo ${ }^{13}$.

Este artigo tem como objetivo refletir sobre o Programa Nacional de Reorientação da Formação Profissional em Saúde (PRÓ-Saúde) articulado ao Programa de Educação pelo Trabalho para a Saúde (PET-Saúde) como cenário mobilizador para a adoção da educação interprofissional, a partir das potências e desafios identificados na IES participantes do PROPET.

\section{Educação interprofissional: uma breve incursão}

Como proposta de formação, a educação interprofissional vem sendo discutida nos últimos trinta anos, especialmente nos Estados Unidos e Europa, com o intuito de estimular o aprimoramento do cuidado em saúde por meio do trabalho em equipe. Os princípios da educação interprofissional se aplicam tanto para a graduação das diferentes profissões de saúde como para a educação permanente dos profissionais componentes de uma equipe de trabalho ${ }^{14}$.

Inicialmente, foi definida como:

Proposta onde duas ou mais profissões aprendem juntas sobre o trabalho conjunto e sobre as especificidades de cada uma, na melhoria da qualidade no cuidado ao paciente. ${ }^{15}$ (p. 460)

Estilo de educação que prioriza o trabalho em equipe, a interdisciplinaridade e o compromisso com a integração das ações que deve ser alcançado com um amplo reconhecimento e respeito às especificidades de cada profissão. ${ }^{16}$ (p.7)

Com a ampliação do debate, outros conceitos começam a ser elaborados com o objetivo de ampliar a compreensão da EIP e evidenciar aspectos importantes que permeiam a efetivação dessa abordagem. Exemplo desses esforços são as revisões sistemáticas que trazem importantes contribuições, a partir da colaboração, comunicação e da centralidade do usuário como pontos centrais da EIP.

Uma intervenção em que os membros de mais de uma profissão da saúde ou assistência social, ou ambos, aprendem em conjunto, de forma interativa, com o propósito explícito de melhorar a colaboração interprofissional ou a saúde/bem-estar de pacientes/clientes, ou ambos. ${ }^{17}$ (p. 5)

Estas concepções permitem reconhecer a possibilidade de inverter a lógica mais usual de pensar a formação em saúde - cada profissão pensada e discutida em si - descortinando espaços e cenários para a incorporação da perspectiva do interprofissionalismo, percebendo que as diferentes áreas profissionais podem constituir um campo mais integrador de práticas de atenção à saúde.

Gyamarti ${ }^{18}$, Casto e Julia ${ }^{19}$, Carpenter $^{20}$ e Freeth ${ }^{21}$, por sua vez, ressaltam que o inteprofissionalismo abrange comunicação entre as diferentes profissões, como forma de assegurar uma atenção integral, opondo-se ao reducionismo e fragmentação da visão de uma profissão isolada.

Nessa perspectiva, há a necessidade de desenvolvimento da competência colaborativa para que o trabalho em equipe possa se efetivar e a EIP se apresente como capaz de reforçar essas competências ${ }^{22}$. Vale ressaltar, ainda, que esses esforços não devem estar centrados apenas na Universidade, e que suas bases teóricas e metodológicas devem subsidiar mudanças/melhorias também nos serviços de saúde ${ }^{23}$.

A EIP vem se apresentando internacionalmente como uma abordagem sólida, e a maior parte das investigações mostra impacto positivo, com evidências de satisfação dos estudantes, dos pacientes e da melhoria de prestação de serviços, como estudo no qual avaliaram-se 21 publicações identificando potencialidades e fragilidades na efetivação da EIP24. 
Outro levantamento, que analisou seis estudos sobre os efeitos da EIP na prática profissional e na melhoria do cuidado, conclui que, embora tenham sido encontrados resultados positivos, não foi possível afirmar a efetividade da EIP em virtude: do pequeno número de publicações incluídas no estudo, da heterogeneidade das amostras e dos métodos utilizados ${ }^{25}$.

No Brasil, estudos realizados por Willians et al. ${ }^{26}$ e Aguilar-da-Silva et al. ${ }^{27}$ trazem pistas promissoras a respeito das percepções e atitudes interprofissionais, sugerindo um impacto positivo a longo prazo, bem como a abertura dos estudantes para a aprendizagem compartilhada.

Rossit et al. ${ }^{12}$, também no contexto brasileiro, em um estudo com egressos de cursos de saúde formados em um currículo baseado na EIP, afirmam que a aprendizagem da prática colaborativa implica: a análise do contexto, o planejamento, a intervenção propriamente dita e a avaliação das ações de saúde de forma compartilhada.

Essa efetividade, considerada como essencial para melhorar a qualidade do cuidado em saúde, é influenciada pelas atitudes dos profissionais em relação à sua própria profissão e aos outros grupos profissionais ${ }^{28}$. Sobre esse aspecto, a literatura debate, como ponto importante, o período em que as atividades de EIP são introduzidas no processo de formação, com vistas a mobilizar atitudes positivas dos estudantes em relação à sua própria profissão e dos outros grupos profissionais.

McNair ${ }^{15}$, por exemplo, descrevem um estudo-piloto de EIP para estudantes de profissões da área da saúde (medicina, enfermagem, fisioterapia e farmácia), realizado na zona rural de Victoria, Austrália, entre 2001 e 2003. A experiência do estágio melhorou as habilidades de autorrelato e conhecimentos no trabalho em equipe, e apoiou a "crença" dos estudantes quanto ao valor da prática interprofissional.

Outro estudo, que relata uma intervenção, baseada na EIP, envolvendo estudantes do $1^{\circ}$ ano de graduação em medicina, fisioterapia, enfermagem e terapia ocupacional, constrói dados qualitativos que mostram o aumento da confiança dos alunos na sua própria identidade profissional, que os ajudou a valorizar as diferenças, tornando-os melhor preparados para a atuação clínica. Os achados corroboram com o debate em torno da necessidade de se inserir a EIP desde o início da formação dos alunos ${ }^{29}$.

Além do período de introdução de iniciativas da EIP, a literatura também discute amplamente os métodos de ensino e aprendizagem mais adotados e suas potencialidades na efetividade da EIP. Goelen et al. ${ }^{30}$ desenvolveram uma investigação que teve como objetivo avaliar a melhoria de atitudes para a colaboração interprofissional de estudantes da área da saúde em um módulo de aprendizagem baseada em problemas (PBL), utilizando pacientes reais como desencadeadores de integração curricular para graduandos em medicina, enfermagem e fisioterapia. No grupo de intervenção, melhorias estatisticamente significativas puderam ser identificadas nas atitudes gerais dos estudantes do gênero masculino e nas atitudes relativas à competência e autonomia dos indivíduos na própria profissão, no grupo como um todo.

Bridges et al. ${ }^{31}$ apresentaram uma investigação sobre três modelos para formação interprofissional. O primeiro tratou de uma proposta curricular enfatizando: as habilidades didáticas, a aprendizagem e o conhecimento das profissões necessárias à formação interprofissional. O segundo, de uma experiência baseada na comunidade, e demonstrou como as colaborações interprofissionais influenciam o serviço e a qualidade do atendimento aos pacientes. O terceiro, de uma atividade de simulação interprofissional, que demonstrou como esta experiência pode desenvolver habilidades de comunicação e liderança.

No entanto, Olson e Bialocerkowsk ${ }^{32}$ chamam a atenção - numa revisão sistemática que buscou identificar a melhor abordagem de EIP para a graduação na área da saúde - para os aspectos mais adequados para proporcionar experiências de aprendizagem ideais. Os resultados dos estudos revisados indicam que a EIP funciona, mas a compreensão do que funciona, para quem e em que circunstâncias é limitada. As autoras defendem a reconceituação da EIP como processo de socialização interprofissional dentro de um sistema, em vez de intervenções transferíveis; e propõem uma agenda de pesquisa que priorize abordagens metodológicas que permitam compreender as complexidades desses processos. Nesse sentido, a EIP precisa ser pensada na realidade em que vai ser adotada, buscando formas, métodos e parceiros que possam avançar na efetividade dos princípios da interprofissionalidade. 


\section{A construção da pesquisa}

O campo empírico do presente estudo foi constituído por cento e vinte Relatórios Técnicos Anuais (RTA) de Projetos PROPET e 119 Relatórios de Autoavaliação (RAV), os quais foram preenchidos pela própria IES participante. Além disto, foram analisados os 99 Relatórios de Acompanhamento (RA) realizados por assessores do Ministério da Saúde durante visitas in loco a cada IES.

Os três instrumentos foram preenchidos por meio do FORMSUS. Nos RTA, realizados no final de 2013, as IES descreviam como estavam sendo desenvolvidas suas ações, destacando avanços e fragilidades. Os RAV, elaborados pelos coordenadores dos projetos aprovados, juntamente com as Comissões Gestoras Locais (CGL), expressaram a autoavaliação do processo de implantação e desenvolvimento das ações em 2013, frente às diretrizes apontadas no edital. Estes RAV buscavam conhecer as percepções das equipes sobre o trabalho que desenvolveram no âmbito do PROPET, avaliando cinco categorias centrais: Comissão de gestão e Acompanhamento local (Espaço de pactuação, negociação e deliberação), Integração ensino/serviço (Educação pelo trabalho, interprofissionalidade e pesquisa), Reorientação do eixo da formação em Saúde, Sustentabilidade e Mudanças nas práticas de cuidado.

Os RA elaborados a partir de visitas de acompanhamento e interlocução crítica, realizadas de junho a dezembro de 2013, abrangem os registros de avaliadores dos projetos PROPET, os quais compõem a Comissão Assessora Nacional ao PRÓ-Saúde e PET-Saúde, orientado pelas mesmas categorias já referidas no RA, mas de forma descritiva.

O acesso aos relatórios foi possível por meio da disponibilização dos mesmos pela SGTES na plataforma do FORMSUS.

A análise dos dados compreendeu uma sistematização quantitativa e uma análise de conteúdo, do tipo temática, na busca dos sentidos atribuídos às atividades interprofissionais no âmbito dos Projetos PROPET ${ }^{33}$.

As seguintes categorias temáticas emergiram nos RTA, RAV e RAA: (1) concepções e práticas na educação interprofissional: multi ou inter?; e (2) EIP e PROPET: desvelando potências.

\section{Educação interprofissional: aprendendo com os projetos PROPET}

\section{Concepções e práticas na educação interprofissional: multi ou inter?}

A discussão sobre educação interprofissional vem ganhando espaço no âmbito dos programas PróSaúde e PET-Saúde, desde 2011, estimulando instituições formadoras a adotarem estratégias baseadas nessa abordagem, tendo em vista sua capacidade de dar respostas a muitos problemas vivenciados no âmbito do processo de formação em saúde ${ }^{5,34}$.

No entanto, refletir sobre as bases teóricas e metodológicas que embasam a educação interprofissional, desvela a necessidade de se dar destaque à confusão conceitual que ora remete à multiprofissionalidade ora à multidisciplinaridade. Não se trata apenas de uma preocupação semântica, mas sim, de esforços no sentido de se compreender a lógica que sustenta a aprendizagem efetivamente compartilhada e colaborativa ${ }^{35}$.

O uso do radical 'disciplinar' se refere a campo ou núcleo do conhecimento ou de estudo, enquanto o 'profissional', a campos ou núcleos de práticas. Associada a esses radicais, há a adoção dos prefixos 'inter' e 'multi', que traduz o nível ou intencionalidade de articulação/interação entre diferentes áreas do conhecimento ou de práticas ${ }^{36}$.

A perspectiva 'multidisciplinar' e 'multiprofissional' aponta para relações independentes entre diferentes áreas do conhecimento ou de práticas, respectivamente. Já a lógica 'interdisciplinar' ou 'interprofissional' está associada à ideia de que diferentes campos do conhecimento e de práticas interagem entre si, de forma colaborativa ${ }^{37}$.

Merece destaque, também, que, não raro, os termos interdisciplinar e interprofissional são utilizados como sinônimos, o que é um equívoco. A interdisciplinaridade pode ser uma ferramenta da 
formação de uma única categoria profissional. Por exemplo, a interação de diferentes disciplinas ou áreas de estudo na formação de uma categoria profissional, a fim de ampliar a compreensão sobre determinado fenômeno. A interprofissionalidade, por sua vez, aponta para a articulação intencional e colaborativa entre diferentes profissões, tendo como resultado ações mais resolutivas e integrais. Assim, a interdisciplinaridade se configura como importante ferramenta da formação interprofissional, mas não devem ser adotadas como sinônimos ${ }^{35}$.

O relatório autoavaliativo aponta importantes aspectos que, por sua vez, demandam a busca por maior detalhamento, presentes nos relatórios técnicos e de acompanhamento. A Tabela 1 traz a percepção dos coordenadores sobre a adoção da interdisciplinaridade e da educação interprofissional na formação dos tutores e preceptores e nas pesquisas desenvolvidas pelas IES.

Tabela 1. Autoavaliação dos coordenadores do PROPET saúde em relação à educação interprofissional e a interdisciplinaridade, julho 2013.

\begin{tabular}{|c|c|c|c|c|}
\hline \multirow{2}{*}{ Respostas } & \multicolumn{2}{|c|}{$\begin{array}{l}\text { Formação de tutores e preceptores } \\
\text { interprofissionais e interdisciplinares }\end{array}$} & \multicolumn{2}{|c|}{$\begin{array}{l}\text { Pesquisas contribuem para a formação } \\
\text { interdisciplinar e interprofissional }\end{array}$} \\
\hline & $\mathrm{N}^{\circ}$ & $\%$ & $\mathrm{~N}^{\circ}$ & $\%$ \\
\hline A - Acontece regularmente & 54 & 41,5 & 104 & 80 \\
\hline B - Acontece parcialmente & 63 & 48,5 & 16 & 12,3 \\
\hline C - Não acontece & 10 & 7,7 & 7 & 5,4 \\
\hline D - Não foi previsto & 1 & 0,8 & 1 & 0,8 \\
\hline NF - não responderam & 2 & 1,5 & 2 & 1,5 \\
\hline
\end{tabular}

Fonte: Relatório Autoavaliativo - FORMSUS

Um primeiro olhar permite afirmar que as IES consideram que vêm adotando, em sua grande maioria, a educação interprofissional e a interdisciplinaridade como instrumentos para a formação dos tutores e preceptores e para o desenvolvimento das pesquisas. É importante salientar que essas respostas podem estar se referindo muito mais a uma perspectiva multiprofissional/multidisciplinar do que à compreensão de 'inter'. As realidades das IES que participam do PROPET, nos instrumentos pesquisados, evidenciam a confusão conceitual (inter e multi), podendo ser compreendida como uma limitação para a adoção de estratégias mais sólidas, na direção da EIP.

Embora pareça óbvio essa diferenciação, muitas vezes, os termos são utilizados de forma indiscriminada, o que justifica a preocupação em delimitar as diferenças e, assim, assegurar que os princípios orientadores da perspectiva interprofissional sejam adotados de uma forma muito clara. Essa confusão conceitual é uma dimensão importante que aparece quando as IES detalham as iniciativas das atividades interprofissionais, impulsionadas pelo PROPET.

“Dentre as atividades interdisciplinares desenvolvidas, destacam-se: Realização de Jornada; Disciplinas interdisciplinares em saúde; Ações multiprofissionais de Saúde semestrais na comunidade; Problematizações interdisciplinares em saúde nos cenários de prática e estágios". (IES 2)

"Os treinamentos multiprofissionais para coleta de dados...". (IES 28)

Os pontos trazidos pelas IES apontam que a materialização das ações fundamenta-se: na busca de possibilitar a aprendizagem compartilhada, a interação entre os diferentes atores na discussão de cenários reais e suas necessidades. Esses pontos apresentam muita aderência com a educação interprofissional, no entanto são apresentados como iniciativas interdisciplinares ou multiprofissionais. 
Outra dimensão observada nos relatos das IES é a pouca clareza ou descrição breve das ações efetivadas na perspectiva da EIP, o que pode apontar para a inexistência de iniciativas ou dificuldades na execução de propostas interprofissionais.

"O trabalho é totalmente multiprofissional e integrativo, posto que é baseado no âmbito da promoção e prevenção. Há nove cursos envolvidos, sempre mesclados no contexto de cada grupo de trabalho". (IES 34)

“No que diz respeito à definição de problemas, objetos e objetivos de pesquisa estão sendo realizadas atividades interdisciplinares, envolvendo tutores, preceptores e alunos de todos os cursos envolvidos. Além disso, estão sendo criados campos de estágio supervisionados para alunos de alguns cursos de graduação". (IES 37)

Por outro lado, os relatórios de acompanhamento (RAA) dos projetos PROPET também trazem os desafios, da realidade atual, frente aos cenários políticos e institucionais complexos, e, muitas vezes, tensos, para a adoção da EIP. É importante que esses aspectos sejam discutidos como forma de se refletir sobre as atuais políticas e planejar estratégias capazes de inserir com mais força a EIP enquanto abordagem importante no cenário de reorientação da formação profissional em saúde.

O enfrentamento das dificuldades trazidas nos relatórios aponta para dimensões distintas, como: o apoio institucional, mudanças na lógica do processo de formação e mudanças atitudinais ${ }^{38}$. É bem verdade que não são questões de fácil superação, mas o PROPET tem demonstrado boa capacidade de mudanças nos contextos das IES que participam dessa política ${ }^{39,40}$.

“Organizam os bolsistas de forma diferente, fazem reuniões coletivas, na qual fazem discussão de artigos juntos, cada um prepara um sobre sua área. Os projetos de pesquisa são meio coletivos também, mas depois eles subdividem". (IES 8)

Os trechos evidenciam importantes dificuldades que se constituem barreiras para a EIP: a dificuldade de pensar em ações que envolvam cursos com diferentes desenhos curriculares, distinção entre duração dos cursos; estruturas físicas das universidades; qualificação docente para estimular a aprendizagem e a prática interprofissional e colaborativa, e realidade do trabalho em saúde, fortemente marcado pela divisão e fragmentação dos atos ${ }^{41}$.

Os desafios que se impõem, no contexto do debate da EPI, reforçam a necessidade de se revisitarem: os desenhos curriculares; os programas dos cursos; a qualificação docente; a reorientação das ações de extensão, pesquisa e ensino, de forma a fortalecer a indissociabilidade; a aproximação com a realidade de vida e saúde, e o fortalecimento dessas ações pelo envolvimento de atores de diferentes cursos da área da saúde e das demais áreas ${ }^{42}$.

\section{EIP e PROPET: desvelando potências}

É possível reconhecer que muitas IES trazem uma descrição detalhada de algumas iniciativas e demonstram aproximação com alguns princípios da EIP, destacando um processo de aprendizagem compartilhado e que se fundamenta nas bases teóricas e metodológicas de um novo profissionalismo e do trabalho colaborativo.

"Os alunos dos vários cursos tem desenvolvido as atividades de maneira integrada, cada um reconhecendo as capacidades e os limites de atuação de sua formação e entendendo que o trabalho integrado beneficia o serviço e a clientela". (IES 3)

“Na medida em que o PET SAÚDE agrega estudantes e profissionais de diferentes cursos da área da saúde em um projeto de trabalho e de pesquisa, todas as atividades são discutidas a partir de diferentes perspectivas. Isto permite que, neste momento, o aluno vivencie uma 
experiência de trabalho inter e multidisciplinar durante o curso de graduação. Este intercâmbio faz com que os alunos tragam para a unidade de saúde, um novo olhar para as atividades em desenvolvimento, como também levam para a graduação a vivência na unidade e o lidar com a comunidade". (IES 42)

Os trechos dos relatórios apontam que o PROPET vem estimulando o enfrentamento de uma forte barreira para a EIP: a construção sócio-histórica da identidade profissional. As categorias profissionais, buscando construir uma identidade socialmente legitimada, estabelecem limites profissionais a partir de um corpo de conhecimentos que lhes atribui relevância social ${ }^{43}$. As profissões, por meio de seus instrumentos legais, buscam construir limites profissionais muito fortes, dificultando sobremaneira: a abertura para tomada de decisões compartilhadas, o gerenciamento de conflitos, para o reconhecimento da importância do papel dos outros profissionais no contexto do trabalho coletivo em saúde.

Nesse sentido, as experiências trazidas pelas IES demonstram o importante impacto do PROPET como política capaz de inserir e fortalecer o debate da EIP no cenário nacional. As instituições já trazem aspectos que evidenciam avanços na superação do modelo tradicional de formação que baliza os silos profissionais. A perspectiva apontada avança no sentido de superar as tribos acadêmicas, e (re) situa o usuário como ator central da dinâmica da produção dos serviços de saúde.

As IES afirmam que a interação possibilitada pelo PROPET permite uma maior visão do contexto em que os alunos estão inseridos, e assegura que profissionais e alunos conheçam outras formas de ver e agir sobre o mundo e suas necessidades ${ }^{44}$. A dinâmica apresentada nos relatórios sustenta 0 argumento de que iniciativas dessa natureza contribuem para a formação de profissionais mais aptos ao trabalho colaborativo no âmbito das equipes de saúde.

Essas evidências, porém, demonstram a necessidade de se ampliarem os efeitos do PROPET para todos os alunos e cursos da área da saúde, construindo uma nova cultura de profissionalismo no âmbito das IES, com impactos em todos os sujeitos envolvidos.

"São estudantes de diferentes anos de formação e de diferentes carreiras, os preceptores também possuem diferentes formações, o que foi relatado como de extrema importância para o processo de trabalho e para a construção do conhecimento. Tal dinâmica de trabalho possibilita, segundo as avaliações dos preceptores e alunos, a geração de aprendizados diversos daqueles obtidos apenas nas aulas teóricas, além de propiciar um envolvimento, vínculo e por parte de muitos preceptores o desejo pela especialização ou pela pós-graduação". (IES 93)

\footnotetext{
"Este processo tem acontecido e entendemos que a simples implicação dos tutores em prol da execução de atividades conjuntas (interdepartamental) já tem sido um enriquecimento no sentido da multiprofissionalidade, visto que antes da existência destes projetos isto não ocorria. Os demais projetos, por serem uniprofissionais, ainda seguem uma linha de execução de atividades ligadas ao núcleo. Contudo, os campos de práticas coincidem, fazendo com que haja alunos de diferentes cursos em períodos semelhantes nos serviços, proporcionando, minimamente, encontro entre eles". (IES 83)
}

Os relatos têm mostrado que o direcionamento dado às ações adotadas pelas IES investem em competências colaborativas, incentivam o rompimento de relações hierárquicas, a reflexão sobre a relevância do trabalho em equipe, competências relevantes no contexto da colaboração e das relações interpessoais, corroborando com experiências trazidas pela literatura ${ }^{45}$.

Muitas instituições no mundo têm desprendido esforços no sentido de descreverem as competências centrais da educação interprofissional, que são pensadas, na maioria das vezes, em cenários da prática clínica ${ }^{46,47}$. Embora sejam contribuições de muita relevância, seria um equívoco adotar essas competências como normas, na medida em que há a necessidade de se rediscutirem as EIP e seus desafios nos diferentes cenários da educação dos profissionais de saúde. 
É possível identificar a potência do PROPET, trazida pelos relatórios de acompanhamento, no sentido de: se conhecer com maior clareza o papel do outro na equipe de saúde; respeitar a importância dos demais profissionais; melhorar a comunicação na discussão dos problemas e na busca de respostas, de forma coletiva; incorporar a compreensão de interdependência entre os membros de uma equipe de saúde; saber conduzir conflitos e tolerar as diferenças entre os profissionais ${ }^{22}$.

\section{Considerações finais}

O PROPET tem uma história significativa de incentivos às mudanças curriculares em diversas IES, em todo o país, configurando-se como importante política que induz a ampliação dos apoios institucionais em torno de temas centrais nos processos de reorientação da formação em saúde. É bem verdade que um processo de mudança, que aponte para mudanças em atitudes historicamente legitimadas, não se configura como tarefa simples. Mas é preciso compreender que a EIP se apresenta como abordagem potente para mudanças tanto nas realidades de produção dos serviços de saúde, quanto no contexto da formação profissional em saúde. Seus princípios se articulam com os propósitos de se avançar na perspectiva da integralidade da atenção e fortalecer a lógica do trabalho em equipe centrado nas necessidades dos usuários, em suas dimensões individual e coletiva.

A análise produzida neste estudo permite reconhecer que o PROPET vem induzindo novas formas de interação e comunicação entre os cursos envolvidos, e seus atores. Além disso, a política favorece que essa interação, como um exercício do trabalho colaborativo nas equipes de saúde, aconteça nos cenários reais de vida e saúde das pessoas, enfrentando dois importantes pontos nevrálgicos da formação em saúde: a articulação ensino/serviço e a qualificação para o trabalho em equipe.

É preciso reconhecer também que o PROPET precisa encontrar mecanismos para aprofundar o debate da EIP no contexto nacional. As IES, embora apresentem importantes avanços, demonstram a necessidade de suporte para a superação das limitações apresentadas e para garantir a sustentabilidade destes avanços. Dessa forma, a SGTES tem um papel importante no sentido de legitimar no nível macro, por meio das políticas de reorientação da formação profissional em saúde, a EIP como proposta potente na luta pela melhoria da qualidade dos serviços de saúde no âmbito do SUS.

\section{Colaboradores}

Marcelo Viana da Costa participou de todas as etapas da elaboração do artigo: idealização, confecção, padronização e submissão. Karina Pavão Patrício participou da idealização e confecção, com destaque para o levantamento dos dados dos relatórios, elaboração e adequação da metodologia do estudo, análise dos dados, e teve importante participação na submissão do artigo. Maria Chagas Sette Câmara participou da idealização do artigo e teve relevante participação na discussão dos resultados. George Dantas Azevedo participou da idealização do artigo, da revisão do texto e da padronização de acordo com as normas da revista. Sylvia Helena Souza da Silva Batista participou de todas as fases de elaboração do artigo: idealização e confecção, e teve participação importante na elaboração da revisão da literatura, ajustes no texto e elaboração dos resumos.

\section{Referências}

1. Demarzo MMP. Transforming health professionals' education. Lancet. 2011;377(9773):1235. http://dx.doi.org/10.1016/S0140-6736(11)60492-3 
2. Ceccim RB, Feuerwerker LCM. Mudança na graduação das profissões de saúde sob o eixo da integralidade. Cad Saúde Pública. 2004;20(5):1400-10. http://dx.doi. org/10.1590/S0102-311X2004000500036

3. Feuerwerker LCM. Educação na saúde: educação dos profissionais de saúde - um campo de saber e de práticas sociais em construção. Rev Bras Educ Med. 2007;31(1):3-4. http://dx.doi.org/10.1590/S0100-55022007000100001

4. World Health Organization. Learning together to work together for health: report of a WHO Study Group on multiprofessional education of health personnel: the team approach. Geneva: World Health Organization; 1988. (Technical report series, vol. 769).

5. Frenk J, Chen L, Bhutta ZA, Cohen J, Crisp N, Evans T et al. Health professionals for a new century: transforming education to strengthen health systems in an interdependent world. Lancet. 2010;376(9756):1923-58. http://dx.doi.org/10.1016/S01406736(10)61854-5

6. Ceccim RB. Estado, sociedade e formação profissional em saúde: contradições e desafios em 20 anos de SUS. Cad Saúde Pública. 2009;25(10):2299-300. http://dx.doi. org/10.1590/S0102-311X2009001000021

7. Rossoni E, Lampert J. Formação de profissionais para o Sistema Único de Saúde e as diretrizes curriculares. Bol Saúde. 2004;18(1):87-98.

8. Ferreira JR, Cury GC, Campos FE, Haddad AE, Gusso GDF. A construção de parcerias como estratégia para o sucesso do Pró-Saúde. Cad ABEM. 2007;3:53-61.

9. Crisp N, Chen L. Global supply of health professionals. N Engl J Med. 2014;370(10):950-7. http://dx.doi.org/10.1056/NEJMra1111610

10. Weller J. Shedding new light on tribalism in health care. Med Educ. 2012;46(2):134-6. http://dx.doi.org/10.1111/j.1365-2923.2011.04178.x

11. Weller J, Boyd M, Cumin D. Teams, tribes and patient safety: overcoming barriers to effective teamwork in healthcare. Postgrad Med J. 2014;90(1061):149-54. http://dx.doi. org/10.1136/postgradmedj-2012-131168

12. Rossit RAS, Batista SH, Batista NA. Formação para a integralidade do cuidado: potencialidades de um projeto interprofissional. Rev Int Humanid Med. 2014;3(1):55-6.

13. Peduzzi M, Norman IJ, Germani AC, Silva JA, Souza GC. Educação interprofissional: formação de profissionais de saúde para o trabalho em equipe com foco nos usuários. Rev Esc Enferm USP. 2013;47(4):977-83. http://dx.doi.org/10.1590/S0080623420130000400029

14. Barr H. Interprofessional education: today, yesterday and tomorrow. London: Learning and Teaching Support Network; 2005.

15. McNair RP. The case for educating health care students in professionalism as the core content of interprofessional education. Med Educ. 2005;39(5):456-64. http://dx.doi. org/10.1111/j.1365-2929.2005.02116.x

16. Barr H. Interprofessional education: today, yesterday and tomorrow. London: Learning and Teaching Support Network; 2002.

17. Reeves S, Perrier L, Goldman J, Freeth D, Zwarenstein M. Interprofessional education: effects on professional practice and healthcare outcomes (update). Cochrane Database Syst Rev. 2013;3: CD002213. http://dx.doi.org/10.1002/14651858.CD002213.pub3

18. Gyarmati G. The teaching of the professions: an interdisciplinary approach. Higher Educ Rev. 1986;18(2):33-43.

19. Casto RM, Julia MC. Interprofessional care and collaborative practice. Ann Arbor: Brooks/Cole; 1994. 
20. Carpenter J. Doctors and nurses: stereotypes and stereotype change in interprofessional education. J Interprof Care. 1995;9(2):151-61. http://dx.doi.org/

21. Freeth D. Interprofessional learning. Edinburgh: Association for the Study of Medical Education; 2007.

22. Barr H. Competent to collaborate: towards a competency-based model for interprofessional education. J Interprof Care. 1998;12(2):181-7.

23. Wilcock PM, Headrick LA. Interprofessional learning for the improvement of health care: why bother? J Interprof Care. 2000;14(2):111-7. v

24. Hammick M, Freeth D, Koppel I, Reeves S, Barr H. A best evidence systematic review of interprofessional education: BEME Guide no 9. Med Teach. 2007;29(8):735-51. http:// dx.doi.org/10.1080/01421590701682576

25. Reeves S, Zwarenstein M, Goldman J, Barr H, Freeth D, Hammick M et al. Interprofessional education: effects on professional practice and health care outcomes. Cochrane Database Syst Rev. 2008(1):CD002213.

26. Williams L, Marks L, Barnhart R, Epps S. ICF-CY: basis for a conceptual model for interprofessional education. In: Paper presented at the 2011 Annual ASHA Convention; 2011 Nov 17-19; San Diego, CA.

27. Aguilar-da-Silva RH, Scapin LT, Batista NA. Avaliação da formação interprofissional no ensino superior em saúde: aspectos da colaboração e do trabalho em equipe. Avaliação (Campinas). 2011;16(1):165-82. http://dx.doi.org/10.1590/S1414-40772011000100009

28. Hind M, Norman I, Cooper S, Gill E, Hilton R, Judd P et al. Interprofessional perceptions of health care students. J Interprof Care. 2003;17(1):21-34. http://dx.doi. org/10.1080/1356182021000044120

29. Cooper H, Spencer-Dawe E, Mclean E. Beginning the process of teamwork: design, implementation and evaluation of an inter-professional education intervention for first year undergraduate students. J Interprof Care. 2005;19(5):492-508. http://dx.doi. org/10.1080/13561820500215160

30. Goelen G, De Clercq G, Huyghens L, Kerckhofs, E,. Measuring the effect of interprofessional problem-based learning on the attitudes of undergraduate health care students. Med Educ. 2006;40(6):555-61. http://dx.doi.org/10.1111/j.13652929.2006.02478.x

31. Bridges DR, Davidson RA, Odegard PS, Maki IV, Tomkowiak J. Interprofessional collaboration: three best practice models of interprofessional education. Med Educ Online. 2011;16:1-10. http://dx.doi.org/10.3402/meo.v16i0.6035

32. Olson R, Bialocerkowski A. Interprofessional education in allied health: a systematic review. Med Educ. 2014;48(3):236-46. http://dx.doi.org/10.1111/medu.12290

33. Minayo MCS. O desafio do conhecimento: pesquisa wualitativa em Saúde. 12a ed. Rio de Janeiro: Hucitec; 2008.

34. Barr H. Engaging with the global challenge. J Interprof Care. 2011;25(5):319-20. http://dx.doi.org/10.3109/13561820.2011.584831

35. Reeves S, Goldman J, Gilbert J, Tepper J, Silver I, Suter E et al. A scoping review to improve conceptual clarity of interprofessional interventions. J Interprof Care. 2011;25(3):167-74. http://dx.doi.org/10.3109/13561820.2010.529960

36. Furlanetto EC. Interdisciplinaridade: um conhecimento construído nas fronteiras. Int Stud Law Educ. 2011;(8):47-54.

37. Oandasan I, Reeves S. Key elements for interprofessional education. Part 1: the learner, the educator and the learning context. J Interprof Care. 2005;19(Suppl 1):S21-38. http://dx.doi.org/10.1080/13561820500083550 
38. Hoffman SJ, Rosenfield D, Gilbert JHV, Oandasan IF. Student leadership in interprofessional education: benefits, challenges and implications for educators, researchers and policymakers. Med Educ. 2008;42(7):654-61. http://dx.doi.org/10.1111/ j.1365-2923.2008.03042.x

39. Ferreira JR, Campos FE, Haddad AE, Cury G. The challenge of improving health and medical care through undergraduate medical education "PRO-SAUDE". Educ Health (Abingdon). 2007;20(2):75.

40. Haddad AE, Brenelli SL, Cury GC, Puccini RF, Martins MA, Ferreira JR et al. Pró-Saúde e PET-Saúde: a construção da política brasileira de reorientação da formação profissional em saúde. Rev Bras Edu Med. 2012;36(1 supl 1):S3-4.

41. Schmitt $M H$, Gilbert JHV, Brandt BF, Weinstein RS. The coming of age for interprofessional education and practice. Am J Med. 2013;126(4):284-8. http://dx.doi. org/10.1016/j.amjmed.2012.10.015

42. Bennett PN, Gum L, Lindeman I, Lawn S, McAllister S, Richards J et al. Faculty perceptions of interprofessional education. Nurse Educ today. 2011;31(6):571-6. http:// dx.doi.org/10.1016/j.nedt.2010.09.008

43. Freidson E. Professionalism, the third logic: on the practice of knowledge. Chicago: University of Chicago Press; 2001.

44. Barr $\mathrm{H}$, Low $\mathrm{H}$. Introducing interprofessional education. Fareham: Center for The Advancement of Interprofessional Education; 2013.

45. Hosny S, Kamel MH, El-Wazir Y, Gilbert J. Integrating interprofessional education in community-based learning activities: case study. Med Teach. 2013;35(suppl 1):S68-73. http://dx.doi.org/10.3109/0142159X.2013.765550

46. Bellack JP, O'neil EH. Recreating nursing practice for a new century. Recommendations and implications of the Pew Health Professions Commission's final report. Nurs Health Care Perspect. 1999;21(1):14-21.

47. Bentley R, Engelhardt JA, Watzak B. Collaborating to implement interprofessional educational competencies through an international immersion experience. Nurs Educ. 2014;39(2):77-84. http://dx.doi.org/10.1097/NNE.0000000000000022

Costa MV, Patrício KP, Câmara AMCS, Azevedo GD, Batista SHSS. Pro-Salud y PET-Salud como espacios de educación interprofesional. Interface (Botucatu). 2015; 19 Supl 1:709-20.

Este articulo pretende reflexionar sobre el Programa Nacional de Reorientación de la Formación Profesional en Salud (Pro-Salud), vinculado al Programa de Educación en el Trabajo para la Salud (PET-Salud) como marco movilizador para la adopción de la educación interprofesional, a partir de los retos identificados en las instituciones de educación superior (IES) participantes en el PROPET. Se investigó a partir de informes de las IES y de asesores del Ministerio de Salud, utilizando la plataforma FORMSUS. El análisis de datos comprendió la sistematización cuantitativa y el análisis de contenido. Emergieron como temas: "Concepciones y prácticas en la educación interprofesional (EIP): ¿multi o inter?" así como" EIP y PROPET: identificando fortalezas. El análisis mostró que el PROPET viene induciendo nuevas formas de interacción entre los cursos involucrados y sus actores, en entornos reales, encarando puntos neurálgicos de la formación en salud: articulación enseñanza/servicio y cualificación para el trabajo en equipo.

Palabras clave: Educación en salud. Servicios de integración docente assistencial. Políticas públicas de Salud.

Recebido em 27/10/14. Aprovado em 16/03/15. 